\title{
Ralf Blank, »Bitter Ends«. Die letzten Monate des Zweiten Weltkriegs im Ruhrgebiet 1944/45, Essen: Klartext 2015, 364 S., EUR 22,95 \\ [ISBN 978-3-8375-1192-5]
}

Besprochen von John Zimmermann: Potsdam, E-Mail: JohnZimmermann@bundeswehr.org

DOI 10.1515/mgzs-2017-0060

Einmal mehr hat sich Ralf Blank des Endes des Zweiten Weltkrieges in seiner Heimatregion angenommen. Der Fachdienstleiter Wissenschaft, Museen und Archive im Fachbereich Kultur der Stadt Hagen darf angesichts seiner zahlreichen Forschungen und Veröffentlichungen getrost als der Experte für dieses Themengebiet bezeichnet werden. Nicht ohne Grund übernimmt es Richard Overy, einer der herausragenden Historiker zur Militärgeschichte des Zweiten Weltkrieges, mit seinem Vorwort in den alliierten Luftkrieg gegen das Deutsche Reich einzuführen und die dementsprechenden Forschungsfelder $\mathrm{zu}$ skizzieren (S. 9-14). Vielleicht meint Blank deswegen, auf eine Einleitung verzichten zu können, die dem weniger kundigen Lesenden die Arbeit vorstellt und sie in den einschlägigen Forschungsstand einordnet. So geht es vergleichsweise abrupt in medias res; bereits im ersten Satz des ersten Kapitels findet man sich in einem ausführlichen Zitat des Time Magazine vom 23. April 1945 über die »Endkämpfe zwischen deutschen und alliierten Truppen« wieder (S. 17).

Diese Kampfhandlungen des letzten halben Kriegsjahres umreißt Blank auf den folgenden sieben Seiten dann nur rudimentär, ehe er zu seinem eigentlichen Vorhaben gelangt: In vier sehr unterschiedlich umfangreichen Kapiteln widmet er sich der westalliierten Kriegführung aus der Luft gegen das Ruhrgebiet und ihren Konsequenzen. Einer knapp 30-seitigen Darstellung des alliierten Vorgehens samt seinen Hintergründen und der völlig überforderten deutschen Luftverteidigung folgt das Herzstück seiner Arbeit: Auf beinahe 160 Seiten beschreibt er Monat für Monat und äußerst detailliert die unterschiedlichen Luftangriffe auf die verschiedenen Ziele in seiner Heimatregion. Wer also wissen möchte, welche und wie viele Flugzeuge von welchem Verband am 5. Oktober 1944 warum »das nördliche und 
nordöstliche Stadtgebiet von Dortmund bombardiert[en]« (S. 57), wie viele und welche Bomben sie dabei abwarfen, um welche Art von Schaden wo zu erzeugen, der wird bei der Lektüre bestens bedient. In bewundernswerter Akribie hat Blank die Chronologie der Attacken zusammengetragen, die dadurch verursachten Schäden protokolliert und die vom NS-Regime auf den jeweiligen hierarchischen Ebenen dagegen unternommenen Maßnahmen beschrieben. Mindestens so beeindruckend wie die nahezu unablässigen Bomberströme und Abwurfmengen erweisen sich nämlich die Aberzehntausende von mehr oder weniger freiwilligen deutschen Arbeitskräften, Zwangsarbeiterinnen und Zwangsarbeiter sowie Kriegsgefangenen, die von den lokalen Parteifunktionären zu Schanz-, Räum- oder Instandsetzungsunternehmungen eingesetzt wurden. Das NS-Regime verfolgte dabei durchaus »einen doppelten Zweck«: Damit sollten nicht nur »Verteidigungsanlagen geschaffen werden, die tatsächlich geeignet sind, dem feindlichen Vordringen Halt zu gebieten«, sondern die Arbeiten darüber hinaus »der moralischen Rückenstärkung unserer Soldaten an der Front «(S. 75) dienen.

Jede einzelne dieser Erkenntnisse ist freilich nicht neu; sie sind allesamt aus den einschlägigen Forschungen bekannt. Blanks Verdienst ist es vielmehr, die Abläufe in einem komprimierten Raum chronologisch nachvollzogen und in ihren Wirkzusammenhang gesetzt zu haben. Damit erhält dieses umfangreiche Kapitel einen handbuchartigen Charakter, der durch die üppige Bebilderung noch unterstrichen wird. Aufgrund der strukturierenden Chronologie und des gefälligen Schreibstils entsteht trotz der fehlenden Fragestellung in der vorliegenden Arbeit hier ein klares Bild, das zweierlei verdeutlicht:

Erstens hat es sich bei der Verschärfung des westalliierten Luftkrieges gegen das Deutsche Reich in der letzten Phase des Zweiten Weltkrieges keinesfalls um willkürliche Luftschläge eines absolut überlegenen Gegners gegen alles gehandelt, was sich auf deutschem Boden befand oder sich dort bewegte. Im Gegenteil verfolgten die Bomberangriffe eindeutige Ziele, wie sie ihnen der Verfasser auch zuordnet: die »[v]erheerende[n] Flächenangriffe« (S. 80-85, 154-159 und S. 174-190), die Angriffe auf die »Hydrierwerke« (S. 85-89) bzw. die Treibstofflager (S. 130-132 und S. 141-145), die »Transportoffensive« gegen die Infrastruktur und Logistik (S. 89-93, 118f. und S. 170-172) oder die Unterstützung der eigenen Bodentruppen (S. 96-100, 112-118, 146-154 und S. 190-210). Dass im Ergebnis eine Stadt nach der anderen in Trümmer sank, die Infrastruktur zerstört oder doch größtenteils lahmgelegt und in der Summe weite Teile der Zivilbevölkerung zunehmend ihrer Existenzgrundlage beraubt wurden, kann also nicht monokausal den westalliierten Luftangriffen zugeschrieben werden, sondern der Tatsache, dass diese erfolgten, weil weder die politische noch die militärische Führung des »Dritten Reiches«, aber eben auch nicht das Gros der Deutschen insgesamt zur Kapitulation bereit gewesen waren. 
Gleichwohl stellt Blank zweitens fest, dass »[d]er Bombenkrieg auf die Zivilbevölkerung [...] eine traumatische Erfahrung [war]«, mit einer entsprechend hohen "Eindringtiefe in die Mentalität» bis weit in die Nachkriegszeit hinein (S. 210). Dabei habe gerade »[d]as Unheimliche des Bombenkrieges, die Rache oder das Böse, herabstürzend von oben, [...] zu zahlreichen mythischen Erzählungen und Legenden über das Geschehen geführt« (ebd.). Bedauerlich bleibt an dieser Stelle der Verzicht des Autors darauf, die Mitverantwortlichkeit eben dieser Bevölkerung für den Krieg und seine Folgen zu thematisieren.

Dennoch engt Blank mit dieser neuerlich umfassenden und detailreichen Studie die noch immer vorhandenen Forschungslücken zum Ende des Zweiten Weltkrieges im Allgemeinen und zu dem im Ruhrgebiet im Besonderen weiter ein. Entgegen der landläufigen Meinung existieren nämlich diverse Fragen, beileibe nicht nur zum Luftkrieg, deren Beantwortung die zahlreichen, meist schuldbefreienden Mythen zum Kriegsende aufdecken würden. Einige dieser Fragen reißt Blank an und bietet Lösungsmöglichkeiten, wie z.B. die längst überfällige Auswertung der »After Action Reports« US-amerikanischer Truppen in den National Archives (S. 240).

Im Ergebnis hat Ralf Blank eine verdienstvolle Studie vorgelegt, die einmal mehr durch überzeugende historische Feinarbeiten besticht. Gleichwohl bleibt er an vielen Stellen die Belege für seine Aussagen schuldig, insbesondere dort, wo Sachverhalt oder Kontext über das Ruhrgebiet hinausreichen. Wenn er beispielsweise aus dem menschenverachtenden und professionell fragwürdigen Durchhalteaktionismus von führenden Militärs wie Generalfeldmarschall Walter Model schließt, diese Einzelschicksale stünden »für einen auch im höheren Offizierkorps verbreiteten Führerglauben, der ihnen die Gefangennahme und den Wechsel vom Krieg in die Nachkriegszeit als eine Unmöglichkeit erscheinen ließ«(S. 262), dann wünscht man sich hierfür schon Belege. Denn die Einsicht in die Niederlage in diesem Krieg, wenigstens aber seine Nicht-Gewinnbarkeit, wurde vor geraumer Zeit bereits gerade für die höhere und höchste militärische Führung lange vor 1944 nachgewiesen; nur ganz wenige glaubten nach der Niederlage von Stalingrad noch an einen alternativen Ausgang.

Nachhaltig negativ bemerkbar machen sich aber während der Lektüre vor allem die mit der Einleitung fehlende präzise Fragestellung sowie ihr methodischer Ansatz und der nahezu folgerichtig fehlende Schluss, der Blanks Ergebnisse zusammenfassen und in verwertbaren Aussagen bündeln könnte. So bleibt die vorliegende Arbeit eine detailreiche deskriptive Darstellung der letzten Kriegsmonate im Ruhrgebiet, die als Handbuch und »Steinbruch« für andere Forschungen sehr wertvoll ist, als eigenständiges Werk jedoch kaum besticht. Angesichts zusätzlicher formaler Mängel wie einem unvollständigen Literaturverzeichnis, der mitunter nur mäßig gelayouteten Bilder oder der im Fließtext 
fehlenden Nummerierung der Kapitel- und Zwischenüberschriften drängt sich der Eindruck auf, hier sei ein Manuskript mit heißer Nadel druckfertig gemacht worden, um unbedingt zur 70-jährigen Wiederkehr des Kriegsendes zu erscheinen. Dies geht spürbar zu Lasten der Aussagekraft, weil das Potenzial der dargelegten Forschungsleistung ohne erkennbare Notwendigkeit nicht ausreichend ausgeschöpft wurde. So bleibt der Erkenntniswert auf Fachleute beschränkt, die in der Lage sind, die recherchierten Fakten zu deuten und für die eigenen Arbeiten nutzbar zu verwenden. Den interessierten Laien hingegen präsentiert sich eine Flut an Details, die außer in ihrer zeitlichen und räumlichen Begrenzung auf das Ruhrgebiet am Ende des Zweiten Weltkrieges durch keine zusammenhängende analytische Interpretation strukturiert wird. Einen möglichst großen Kreis von Leserinnen und Lesern aber hätte diese Arbeit angesichts ihres Faktenreichtums fraglos verdient. 